\title{
Production of $\mathrm{Ag}$ Nanocubes on a Scale of $0.1 \mathrm{~g}$ per Batch by Protecting the NaHS-Mediated Polyol Synthesis with Argon
}

\author{
Qiang Zhang ${ }^{\dagger} \ddagger$, Claire Cobley ${ }^{\dagger}$, Leslie Au ${ }^{\dagger}$, Maureen McKiernan $\uparrow$, Andrea Schwartz ${ }^{\dagger}$, Long- \\ Ping Wen $\ddagger$, Jingyi Chen $\dagger,{ }^{*}$, and Younan Xia ${ }^{,}{ }^{\star}$ \\ † Department of Biomedical Engineering, Washington University, St. Louis, Missouri 63130 \\ $\ddagger$ Hefei National Laboratory for Physical Science at the Microscale, University of Science and \\ Technology of China, Hefei, Anhui 230027, P. R. China \\ I Department of Chemistry, Washington University, St. Louis, Missouri 63130
}

\begin{abstract}
Gold nanocages synthesized from Ag nanocubes via the galvanic replacement reaction are finding widespread use in a range of applications due to their tunable optical properties. Most of these applications require the use of nanocages with a uniform size and in large quantities. This requirement translates into a demand for scaling up the production of Ag nanocubes with uniform, well-controlled sizes. Here we report such a method based on the modification of NaHS-mediated polyol synthesis with argon protection for fast reduction, which allows for the production of Ag nanocubes on a scale of $0.1 \mathrm{~g}$ per batch. The Ag nanocubes had an edge length tunable from $25 \mathrm{~nm}$ to $45 \mathrm{~nm}$ together with a size variation within $\pm 5 \mathrm{~nm}$. The use of argon protection was the key to the success of this scaleup synthesis, suggesting the importance of controlling oxidative etching during a synthesis.
\end{abstract}

\section{Keywords}

silver nanocubes; polyol synthesis; scale-up production; and surface plasmon resonance

\section{INTRODUTION}

Silver nanoparticles have been extensively studied over the past decades because they exhibit unique surface plasmon resonance (SPR) properties in the visible region that are useful for chemical and biological sensing. ${ }^{1-} 4$ Among various Ag nanoparticles, nanocubes with a single crystalline structure are particularly interesting for their use as templates in the production of Au nanocages that hold great promise for a range of biomedical applications, including optical sensing, imaging contrast enhancement, drug delivery, and photothermal cancer treatment. $5^{-}$ 8 To systematically evaluate the use of Au nanocages in vivo, one needs to produce such nanostructures in relatively large quantities, together with uniform sizes and controllable optical resonance peak positions. For example, the typical injection dosage of Au nanocages for an individual mouse is on the order of $10^{12}$ particles in order to observe signal enhancement for newly developed imaging modalities such as optical coherence tomography (OCT) and photoacoustic tomography (PAT) ${ }^{9}$ or to achieve effective photothermal cancer destruction. 10,11 This translates into the same number of $\mathrm{Ag}$ nanocubes that serve as sacrificial templates during the galvanic replacement reaction.

*Corresponding authors: xia@ biomed.wustl.edu; chenj@ seas.wustl.edu. 
The NaHS-mediated polyol synthesis has been shown to be a rapid reduction route to the production of relatively small Ag nanocubes. ${ }^{12}$ However, the quantity of Ag nanocubes that can be obtained per batch of synthesis is typically on the order of $0.01 \mathrm{~g}$ solid or $2.0 \times 10^{13}$ cubes of $40 \mathrm{~nm}$ in size. This amount is only sufficient for injection into approximately ten mice. To improve the statistics of in vivo studies, one has to deal with a larger number of mice and thus needs to combine Au nanocages derived from many different batches of $\mathrm{Ag}$ nanocubes. This approach can be very tedious and time-consuming. On the other hand, use of different batches of $\mathrm{Ag}$ nanocubes may introduce unwanted variations to the size and quality and further affect the outcome of an in vivo study. Here we provide a solution to this problem by scaling up the production of $\mathrm{Ag}$ nanocubes through the use of a continuous argon flow. By introducing the argon protection, the quantity of $\mathrm{Ag}$ nanocubes per batch was increased from 0.01 to 0.1 $\mathrm{g}$, a ten-fold increase relative to the previous work. The new synthesis also showed much better reproducibility: typically, nine out of ten syntheses yielded high-quality Ag nanocubes.

\section{EXPERIMENTAL SECTION}

\section{Synthesis of Ag nanocubes}

$60 \mathrm{~mL}$ of ethylene glycol (EG, J. T. Baker, Lot No. G32B27) was added into a $250 \mathrm{~mL}$ roundbottom flask and heated in an oil bath at $150{ }^{\circ} \mathrm{C}$ under magnetic stirring with a large, eggshaped Teflon-coated stir bar. After 50 min of pre-heating, a flow of argon was introduced via a glass pipette at a flow rate of $1200 \mathrm{~mL} / \mathrm{min}$, as shown in Figure $1 \mathrm{~A}$. After $10 \mathrm{~min}, 0.7 \mathrm{~mL}$ of a sodium hydrosulfide (NaHS, Aldrich) solution in EG $(3 \mathrm{mM})$ was quickly injected into the pre-heated EG solution, followed by injection of $15 \mathrm{~mL}$ of poly(vinyl pyrrolidone) (PVP, M.W. $\approx 55,000$, Aldrich) solution in EG $(20 \mathrm{mg} / \mathrm{mL})$ and $8 \mathrm{~min}$ later $5 \mathrm{~mL}$ of silver nitrate $\left(\mathrm{AgNO}_{3}\right.$, Aldrich) solution in EG $(48 \mathrm{mg} / \mathrm{mL})$. The reaction flask was capped by a septum with a small opening to allow the gaseous species to escape from the flask. Shortly after the addition of $\mathrm{AgNO}_{3}$, the reaction solution went through four distinct stages of color change from golden yellow to deep red, reddish grey, and then green ocher within about $20 \mathrm{~min}$. The green ocher color indicates the formation of uniform $\mathrm{Ag}$ nanocubes with an edge length around $45 \mathrm{~nm}$. The reaction solution was then quenched by placing the reaction flask in an ice-water bath. Quenching at a slightly earlier point (while the reddish color was still visible) would result in smaller cubes with an edge length of $\sim 35 \mathrm{~nm}$.

For mechanistic studies, we also conducted a set of syntheses and quenched the reaction at early stages when different colors were observed. For comparison, aliquots were also taken at different stages of a synthesis with a glass pipette and quickly added into centrifuge tubes containing acetone pre-cooled to $0{ }^{\circ} \mathrm{C}$. The resultant products were washed with acetone, followed by deionized water to remove excess EG and PVP for characterization and storage. Finally, the $\mathrm{Ag}$ nanocubes were re-dispersed in $10 \mathrm{~mL}$ of water. In a typical synthesis, the final product contained $0.1 \mathrm{~g}$ of solid Ag. The theoretical amount of Ag produced from all the added $\mathrm{AgNO}_{3}$ was $0.15 \mathrm{~g}$. Therefore, the overall yield of the synthesis was $67 \%$. The loss of Ag can be attributed to the following two major sources: $i$ ) a very thin layer of Ag was often observed to plate on the inner wall of the flask during the synthesis; and ii) a small amount of $\mathrm{Ag}$ nanocubes lost with the supernatant during the centrifugation/washing process.

\section{Instrumentation}

The sample for scanning electron microscopy (SEM) was prepared by dropping a few microliters of the product onto a small piece of silicon wafer, followed by drying under ambient conditions. The sample for transmission electron microscopy (TEM) was prepared by replacing the silicon wafer with a carbon-coated copper grid. The SEM images were taken using a field emission microscope (FEI, Nova NanoSEM 230) operated at an accelerating voltage of 10-20 $\mathrm{kV}$. The TEM images were captured using a microscope (FEI G2 Spirit Twin) operated at 120 
$\mathrm{kV}$. The high-resolution TEM images were recorded using a JEOL 2100F electron microscope operated at $200 \mathrm{kV}$. The UV-vis spectra were taken using a Cary 50 spectrophotometer (Palo Alto, CA). The sample for inductively coupled plasma mass spectrometry (ICP-MS, Agilent $7500 \mathrm{CE}$ ) was prepared by dissolving $10 \mu \mathrm{L}$ of the $\mathrm{Ag}$ nanocube suspension with $30 \mu \mathrm{L}$ of concentrated $\mathrm{HNO}_{3}$. The resultant solution was further diluted to a level of $100 \mathrm{ppb}$ for ICPMS analysis.

\section{RESULTS AND DISCUSSION}

The scale-up production of $\mathrm{Ag}$ nanocubes was conducted in a round-bottom flask equipped with a glass pipette to introduce a continuous flow of argon above the reaction solution (see Fig. 1A). The glass pipette was bended to avoid disturbing the surface of the reaction solution. It was not necessary to use a condenser because the reaction temperature $\left(150^{\circ} \mathrm{C}\right)$ was well below the boiling point of ethylene glycol $\left(197^{\circ} \mathrm{C}\right)$ and the reaction was completed within a relative short period of time $(<1.5 \mathrm{~h})$. Similar to a conventional polyol synthesis, ethylene glycol was oxidized by $\mathrm{O}_{2}$ from air to glycolaldehyde in a pre-heating step, ${ }^{13}$ which could then serve as a reductant for $\mathrm{AgNO}_{3}$ :

$$
\begin{aligned}
2 \mathrm{AgNO}_{3} & +\mathrm{HOCH}_{2} \mathrm{CHO} \\
& +\mathrm{H}_{2} \mathrm{O} \rightarrow 2 \mathrm{Ag} \\
& +\mathrm{HOCH}_{2} \mathrm{COOH}+2 \mathrm{HNO}_{3}
\end{aligned}
$$

The pre-heating was typically continued for 50 min under an air atmosphere, and the system was then flushed with argon for 10 min before a trace amount of NaHS was added. It should be pointed out that the same molar ratio of $\mathrm{NaHS}$ to $\mathrm{AgNO}_{3}$ was also used in a small-scale synthesis without argon protection, where Ag nanocubes were observed to form very quickly (typically, within $30 \mathrm{~min}$ ). According to our previous work, the mechanism is based on the formation of highly insoluble $\mathrm{Ag}_{2} \mathrm{~S}$ crystallites, as indicated by a fleeting purple color when $\mathrm{AgNO}_{3}$ was introduced. ${ }^{12,14}$ Silver sulfide is a well-known catalyst for $\mathrm{Ag}$ reduction, which can greatly shorten the timescale for $\mathrm{AgNO}_{3}$ reduction by hundreds of folds, allowing the thermodynamically less favorable, single-crystal, cubo-octahedral seeds to form through a heterogeneous nucleation process.

The NaHS-mediated synthesis has been used with great success in small vials under an air atmosphere to produce $\mathrm{Ag}$ nanocubes on the scale of $0.01 \mathrm{~g}$ per batch. When we tried out this synthesis on a 5-10 times larger scale, the product was dominated by multiple-twinned particles with a more or less spherical shape. In the present work, we were able to generate $0.1 \mathrm{~g}$ of highquality Ag nanocubes in a single run of synthesis by flowing argon over the reaction solution (Fig. 1A). After flushing the system with argon for $10 \mathrm{~min}$, the system was kept under argon protection at a flow rate of $1200 \mathrm{~mL} / \mathrm{min}$ throughout the addition of reagents and the growth of $\mathrm{Ag}$ nanocubes. The argon flow can remove the $\mathrm{NO}_{2}$ generated as a by-product from the decomposition of $\mathrm{NO}_{3^{-}}$, as well as the oxygen present in the reaction system. To confirm the presence of $\mathrm{NO}_{2}$, we allowed the argon to pass through two centrifuge tubes filled with water. One of the tubes (tube A) was placed before the argon entered the reaction system while the other one (tube B) was placed after the argon had passed through the system. We measured the $\mathrm{pH}$ value of the water in these two tubes using a $\mathrm{pH}$ meter. The $\mathrm{pH}$ in tube $\mathrm{B}$ decreased to 5.6, much lower than that in tube $\mathrm{A}(\mathrm{pH}=6.8)$. This result implies that the $\mathrm{NO}_{2}$ could be present in the reaction, which was supposed to react with water to produce $\mathrm{HNO}_{3}$, resulting in the reduction of $\mathrm{pH}$. To rule out the possibility that $\mathrm{NO}_{2}$ may affect the reaction, we replaced the gas inlet and outlet in Figure 1A with glass stoppers after the initial 10 min of argon flow to keep $\mathrm{NO}_{2}$ in the reaction system. Although $\mathrm{NO}_{2}$ was accumulated in the system, no difference was observed for the reaction and its product as compared to the previous setup. This result 
suggests that the replacement of air above the solution that contains oxygen by argon is responsible for the successful operation of this synthesis, rather than the removal of gaseous species generated during the reaction.

Figure 1, B and C, shows SEM and TEM image of a typical product of Ag nanocubes of $45 \pm 5$ $\mathrm{nm}$ in edge length synthesized using the argon protection method. A very small amount of bipyramids $(<4 \%)$ were also present in the product, which evolved from single-twinned seeds. ${ }^{17}$ In this case, $67 \%$ of the added $\mathrm{AgNO}_{3}$ was reduced and converted into $\mathrm{Ag}$ nanocubes and bipyramids. The high-resolution TEM image in Figure 1D, taken from the boxed region in the inset TEM image, shows a well-resolved lattice fringe with a spacing of $2.0 \AA$ parallel to the edges of the cube. This number corresponds to the $\{200\}$ lattice spacing of face-centered cubic silver, suggesting that the cube was enclosed by $\{100\}$ facets. The same pattern of lattice fringe was observed over the entire nanocube, confirming single crystallinity for the nanocube.

The synthesis can be divided into four distinctive stages based on color changes in 20 min after the addition of $\mathrm{AgNO}_{3}$. To study the mechanism, the reaction was terminated at early stages by quickly pouring the hot reaction solution into acetone pre-cooled to $0{ }^{\circ} \mathrm{C}$. Figure 2 shows TEM images of samples obtained for the four different stages: 5, 10, 15, and $20 \mathrm{~min}$ after the addition of $\mathrm{AgNO}_{3}$. The inset shows photograph of the corresponding reaction solution. At $\mathrm{t}=5$ $\mathrm{min}$, the reaction solution was in a golden yellow color, and small single-crystal seeds of $<10$ $\mathrm{nm}$ in size were observed. Note that a few twinned seeds might still exist at this stage which would grow into irregular particles. At $\mathrm{t}=10 \mathrm{~min}$, the reaction became deep red and singlecrystal cubo-octahedral seeds of $\sim 20 \mathrm{~nm}$ in size dominated the sample. Since the polymeric capping agent, PVP, preferentially stabilizes the $\{100\}$ facets, the cubo-octahedral seeds could grow into small cubes of $\sim 26 \mathrm{~nm}$ in edge length after another $5 \mathrm{~min}$ as the reaction solution became reddish grey. At $t=20 \mathrm{~min}$, when the solution appeared green-ocher, nanocubes of $\sim 45$ $\mathrm{nm}$ in edge length were obtained.

In addition to the electron microscopy study, we have also monitored the progress of the reaction using UV-vis spectroscopy. Figure 3A shows a photograph of the as-prepared samples suspended in water. The discrepancy in color as compared to the pictures in Figure 2 can be attributed to the differences in concentration and solvent (water $v s$. ethylene glycol). The corresponding extinction spectra are shown in Figure 3B. The extinction peak caused by SPR red-shifted as the reaction proceeded: $391 \mathrm{~nm}, 400 \mathrm{~nm}, 412 \mathrm{~nm}$, and $450 \mathrm{~nm}$ for $\mathrm{t}=5,10,15$, and $20 \mathrm{~min}$, respectively. This shift of extinction peak was in agreement with the TEM observations of the particle sizes, that is, the SPR peak was red-shifted as the particle size increased. ${ }^{18}$ The spectrum for the sample taken at $\mathrm{t}=20 \mathrm{~min}$ also displayed two additional, relatively weak peaks at $350 \mathrm{~nm}$ and $390 \mathrm{~nm}$, indicating the formation of $\mathrm{Ag}$ nanocubes with sharp corners. For comparison, aliquots were taken at four different stages of a single synthesis and then analyzed by TEM. As shown in Figure 4, similar results were observed compared to the four different parallel reactions.

Oxidation of $\operatorname{Ag}(0)$ to $\operatorname{Ag}(\mathrm{I})$ via oxidative etching has been shown to be a critical factor in determining the yield of Ag nanocubes in a polyol synthesis. ${ }^{15}$, 16 However, we found that if too much oxygen was present during a synthesis, oxidative etching of the small nuclei at early stages could greatly slow down the growth of Ag nanocubes.12 While the amount of oxygen was easier to control in a small-scale system such as a tightly capped, small vial, the large flask needed for a scale-up synthesis might contain too much oxygen in the system. Additionally, it might be possible that oxidative etching can disrupt the formation of $\mathrm{Ag}_{2} \mathrm{~S}$ nanocrystallites that are responsible for catalyzing the reduction of $\mathrm{AgNO}_{3}$. This would also lead to a slow reduction rate and thus multiply twinned particles for the final product. By removing oxygen from the reaction system after the pre-heating step, argon protection offers a highly reproducible route to $\mathrm{Ag}$ nanocubes in large quantities and high quality. Note that 
glycolaldehyde (from the oxidation of ethylene glycol in air) is no longer needed after some Ag nanocrystals have been formed in the solution due to their autocatalytic property.

Alternatively, we can also limit the amount of oxygen present in a reaction system by filling the solution to the neck of the flask, as shown in Figure 5A. After preheating for $50 \mathrm{~min}$ and argon bubbling for $10 \mathrm{~min}$, reagents were added to the EG in the same amounts and in the same sequence as previous syntheses. The reaction flask was then capped by a glass stopper so that no addition oxygen could enter into the reaction system. Figure 5B shows the typical product of this simple setup, where $90 \%$ of the sample was single-crystal nanocubes. It is worthy pointing out that the details of a reaction setup could also affect the quality of the product. For example, when argon was directly bubbled into the solution during the synthesis, small Ag nanocubes and some large twinned particles were found in the product, as shown in Figure 5, $\mathrm{C}$ and $\mathrm{D}$. This is probably due to the inhomogeneity of nucleation when the reaction solution was passed with gas bubbles.

In order to confirm the role of argon, we conducted the synthesis under four different gas atmospheres: oxygen, air, nitrogen, and argon. The amount of oxygen existed in the gas atmosphere strongly affects the yield of the Ag nanocubes (Table 1). When the reaction occurred under oxygen, it became extremely slow and the reaction solution color stayed golden yellow and never went through the dark red stage or reached the green ocher color. Figure 6A shows a typical product that was collected 20 min after $\mathrm{AgNO}_{3}$ was added. Most of the particles appeared to have twinned structures. Similar products with twinned structures were observed at longer reaction times (e.g., $\mathrm{t}=18 \mathrm{~h}$ ). As the amount of oxygen was decreased by conducting the reaction in air, the reaction remained slow and no apparent difference was observed in color as compared to the reaction done in pure oxygen. As shown in Figure 6B, the majority of the particles also existed as twinned particles. This observation suggests that there was still too much oxygen in the air for achieving fast reduction. When the oxygen was completely eliminated by argon or nitrogen after the addition of $\mathrm{AgNO}_{3}$, the reaction became much faster and the distinctive changes in color were visualized as described previously. Figure 6, C and $\mathrm{D}$, shows TEM images of the typical products obtained under nitrogen and argon protection, respectively. The reaction under the argon protection had a better yield of Ag nanocubes than that protected by nitrogen. Generally, argon is a better protection gas than nitrogen because the molecular weight of argon is higher than that of air and thus a better gas for replacing the air (or more appropriately, oxygen) trapped in the reaction system.

\section{Conclusion}

We have successfully modified the NaHS-mediated polyol synthesis with argon flow for routine, scale-up production of $\mathrm{Ag}$ nanocubes. The quantity of $\mathrm{Ag}$ nanocubes could be increased from 0.01 to $0.1 \mathrm{~g}$ for each run of synthesis. With an edge length of $40 \mathrm{~nm}$, the total number of Ag nanocubes contained in a typical batch of sample was on the order of $2.0 \times 10^{14}$, which is enough for generating Au nanocages sufficient for in vivo studies with at least 100 mice. The key to the successful operation of this synthesis is to effectively control oxidative etching involved in the reaction by protecting the system with a flow of argon for fast reduction. As demonstrated in this work, oxygen (from air) is only needed in the preheating stage of a NaHS-mediated polyol synthesis for generating glycolaldehyde (the reductant). After that, it should be removed from the reaction system to prevent oxidative etching and thus speed up the reduction of $\mathrm{AgNO}_{3}$. The improvement in both quantity and quality for $\mathrm{Ag}$ nanocubes is critical for the production of Au nanocages sought for biomedical research and clinical applications. 


\section{Acknowledgments}

This work was supported in part by a 2006 Director's Pioneer Award from the NIH (DP1 OD000798) and startup funds from Washington University in St. Louis. As a visiting student from the University of Science and Technology of China (USTC), Q.Z. was also partially supported by the China Scholarship Council.

\section{REFERENCES AND NOTES}

1. Cao YWC, Jin R, Mirkin CA. Science 2002;297:1536-1540. [PubMed: 12202825]

2. Moore BD, Stevenson L, Watt A, Flitsch S, Turner NJ, Cassidy C, Graham D. Nat Biotechnol 2004;22:1133-1138. [PubMed: 15300259]

3. Wiley B, Sun Y, Xia Y. Acc Chem Res 2007;40:1067-1076. [PubMed: 17616165]

4. Anker JN, Hall WP, Lyandres O, Shah NC, Zhao J, Van Duyne RP. Nat Mater 2008;7:442-453. [PubMed: 18497851]

5. Chen J, Saeki F, Wiley BJ, Cang H, Cobb MJ, Li ZY, Au L, Zhang H, Kimmey MB, Li X, Xia Y. Nano Lett 2005;5:473-477. [PubMed: 15755097]

6. Chen J, Wang D, Xi J, Au L, Siekkinen A, Warsen A, Li ZY, Zhang H, Xia Y, Li X. Nano Lett 2007;7:1318-1322. [PubMed: 17430005]

7. Yang X, Skrabalak SE, Li ZY, Xia Y, Wang LV. Nano Lett 2007;7:3798-3802. [PubMed: 18020475]

8. Skrabalak SE, Chen J, Sun Y, Lu X, Au L, Cobley CM, Xia Y. Acc Chem Res 2008;41:1587-1595. [PubMed: 18570442]

9. Li PC, Wang CRC, Shieh DB, Wei CW, Liao CK, Poe C, Jhan S, Ding AA, Wu YN. Opt Express 2008;16:18605-18615. [PubMed: 19581946]

10. O’Neal DP, Hirsch LR, Halas NJ, Payne JD, West JL. Cancer Lett 2004;209:171-176. [PubMed: 15159019]

11. Lu W, Xiong C, Zhang G, Huang Q, Zhang R, Zhang JZ, Li C. Clin Cancer Res 2009;15:876-886. [PubMed: 19188158]

12. Skrabalak SE, Au L, Li X, Xia Y. Nat Protoc 2007;2:2182-2190. [PubMed: 17853874]

13. Skrabalak SE, Wiley BJ, Kim M, Formo EV, Xia Y. Nano Lett 2008;8:2077-208. [PubMed: 18507481]

14. Siekkinen AR, McLellan JM, Chen J, Xia Y. Chem Phys Lett 2006;432:491-496. [PubMed: 18496589]

15. Wiley B, Herricks T, Sun Y, Xia Y. Nano Lett 2004;4:1733-1739.

16. Taguchi A, Fujii S, Ichimura T, Verma P, Inouye Y, Kawata Satoshi. Chem Phys Lett 2008;462:92_ 95.

17. Wiley BJ, Xiong Y, Li ZY, Yin Y, Xia Y. Nano Lett 2006;6:765-768. [PubMed: 16608280]

18. Im SH, Lee YT, Wiley B, Xia Y. Angew Chem Int Ed 2005;44:2154-2157. 

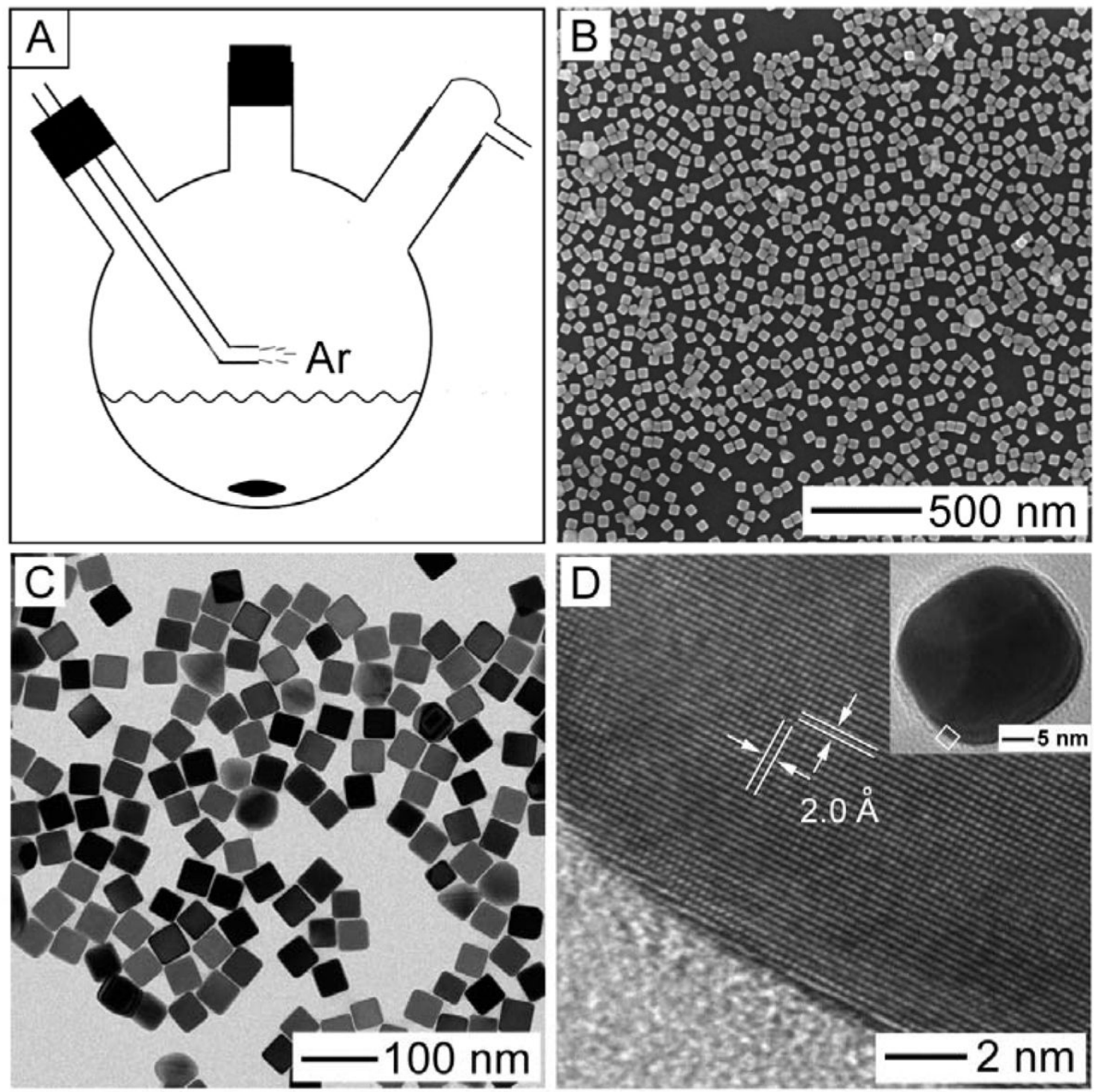

Figure 1.

(A) Schematic illustrating the setup used for synthesizing Ag nanocubes on a scale of $0.1 \mathrm{~g}$ per batch. The key is to modify the NaHS-mediated polyol synthesis with argon protection. (B, C) SEM and TEM images of the as-synthesized Ag nanocubes, which were $\sim 45 \mathrm{~nm}$ in edge length. (D) High-resolution TEM image of the selected area of an individual Ag nanocube shown in the inset. 


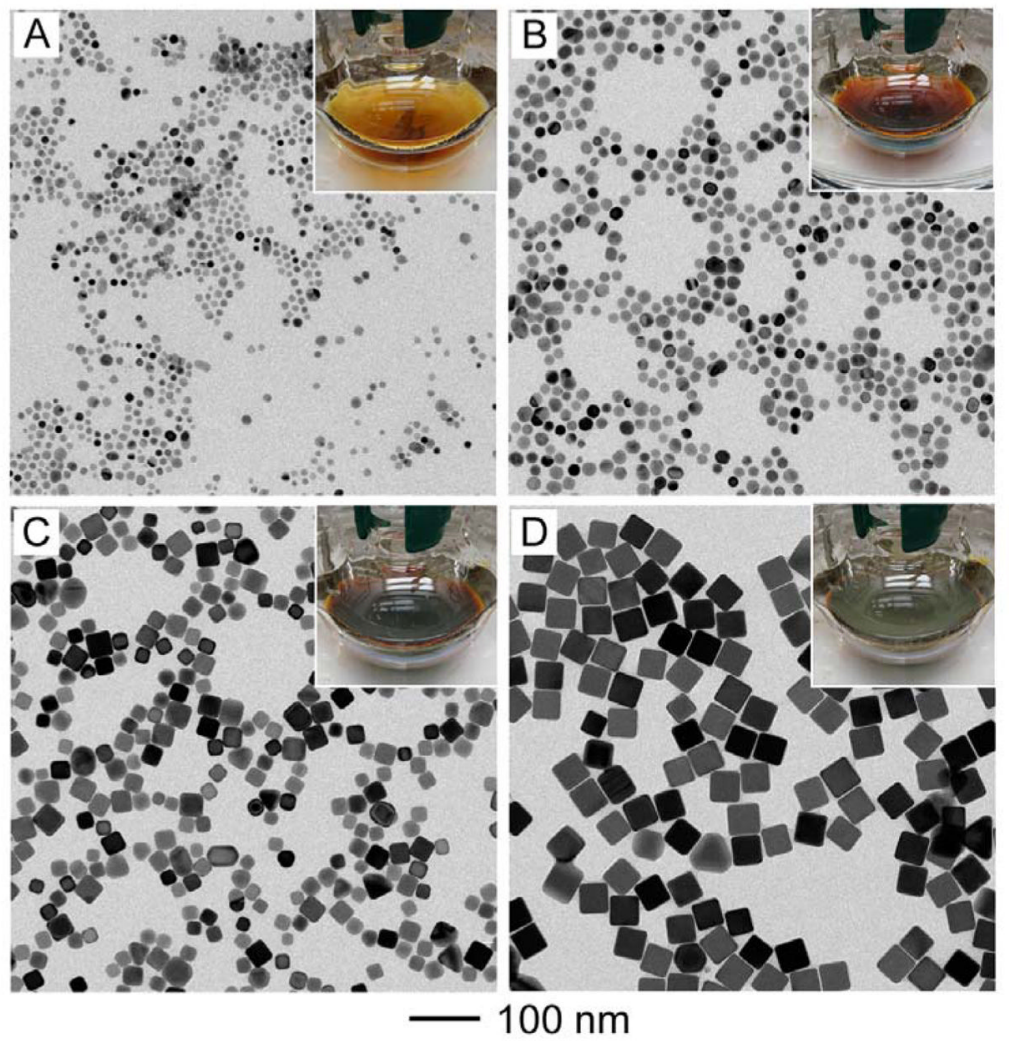

Figure 2.

TEM images of the products obtained from four parallel syntheses under argon, where the reaction was quenched after the $\mathrm{AgNO}_{3}$ solution had been added for: (A) $5 \mathrm{~min}$, (B) $10 \mathrm{~min}$, (C) $15 \mathrm{~min}$, and (D) $20 \mathrm{~min}$. The insets are photographs of the corresponding reaction solutions. The colors of the solutions changed from golden yellow to deep red, reddish grey, and finally green ochre as the reactions proceeded. 

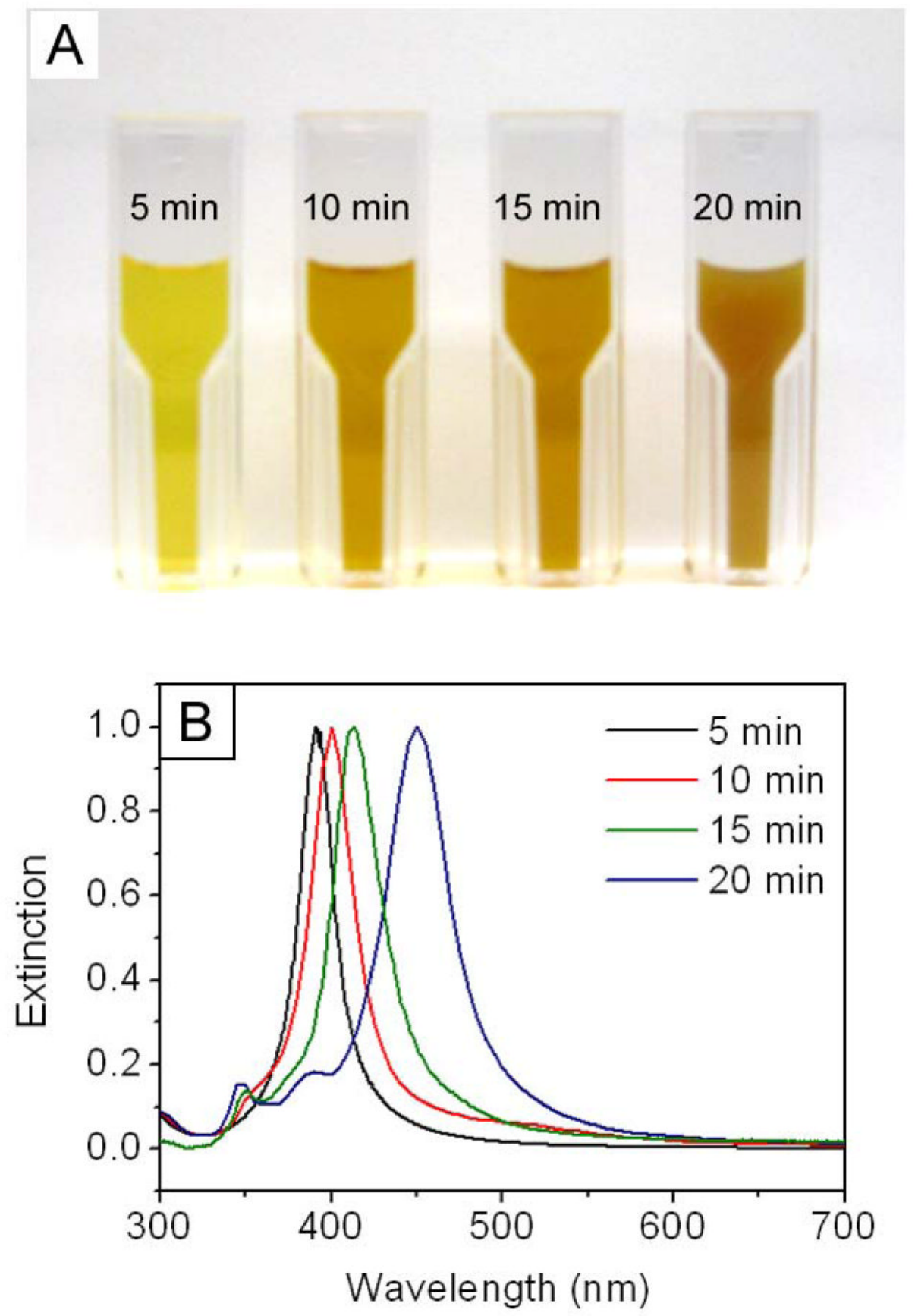

Figure 3.

(A) Photographs of samples obtained from for the parallel syntheses (under argon) that were quenched after the addition of $\mathrm{AgNO}_{3}$ solution for different periods of time: 5, 10, 15, and 20 min. (B) UV-vis spectra taken from the solutions shown in (A). 

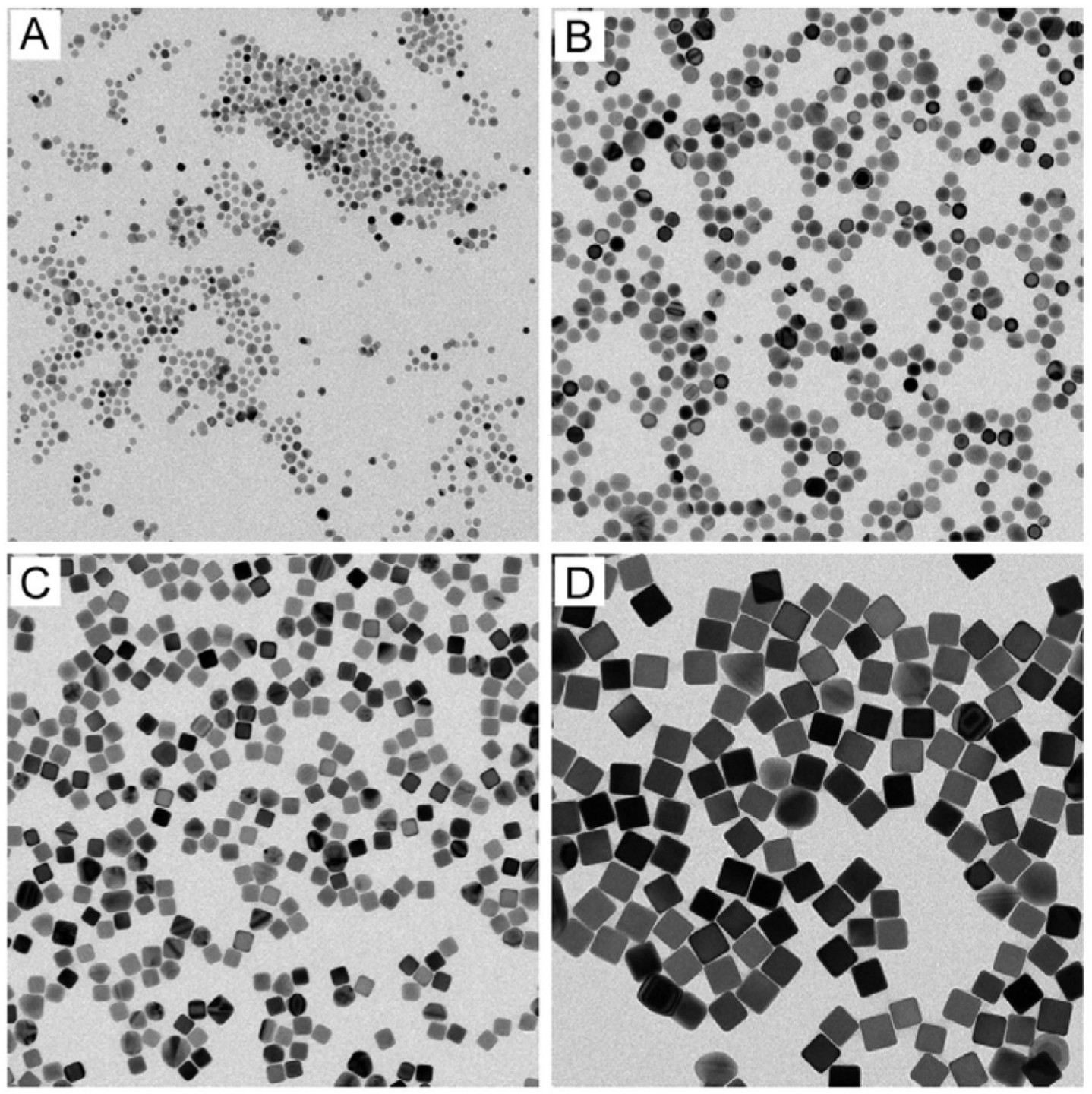

\section{$100 \mathrm{~nm}$}

Figure 4.

TEM images of four aliquots taken from the same synthesis (under argon) after the $\mathrm{AgNO}_{3}$ solution had been added for different periods of time: (A) $5 \mathrm{~min}$, (B) $10 \mathrm{~min}$, (C) $15 \mathrm{~min}$, and (D) $20 \mathrm{~min}$. 

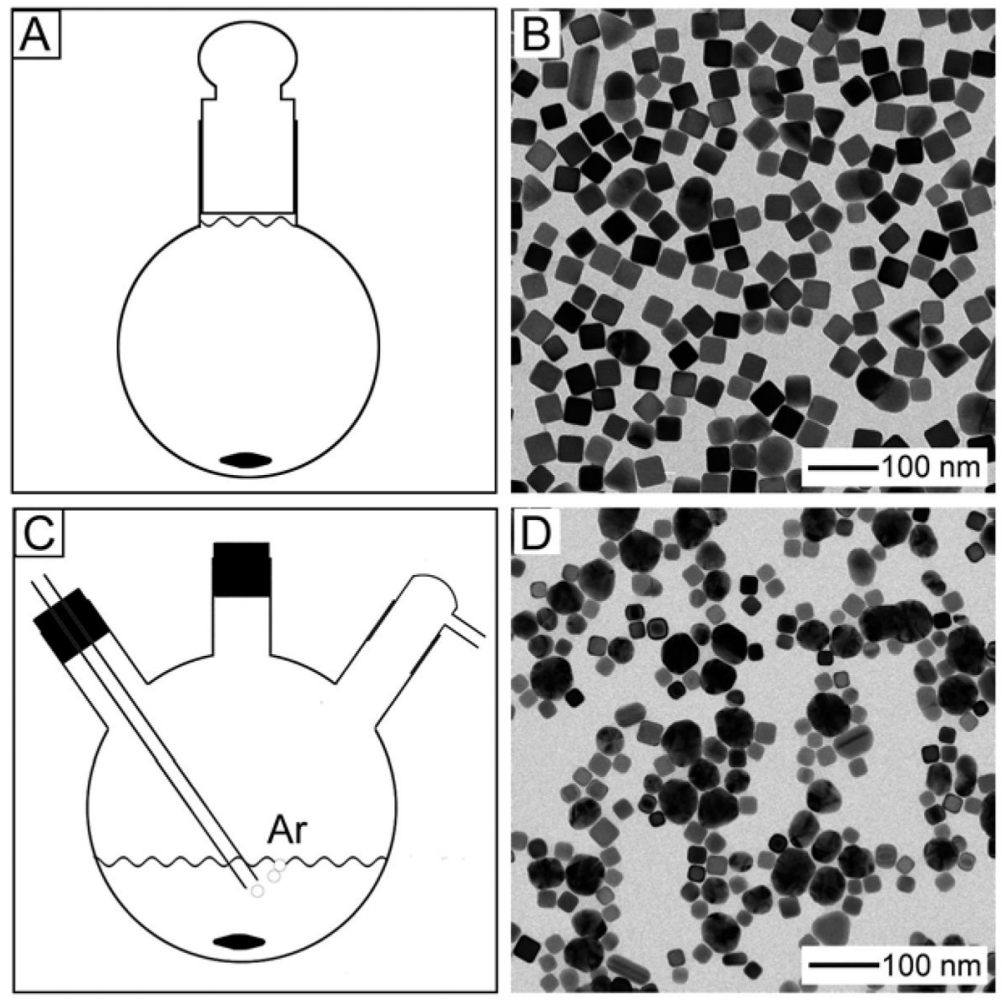

Figure 5.

(A) Schematic of the experimental setup, in which the reaction solution is completely filled to the top of the flask to have limited access of oxygen from the air. (B) Typical TEM image of the product obtained using this setup, which contained $90 \%$ of the single-crystal nanocubes in the product. (C) Schematic of the experimental setup, in which the argon was bubbling through the reaction solution. (D) Typical TEM image of the product obtained using this setup, which contained a mix of single-crystal nanocubes and multiply twinned particles. 


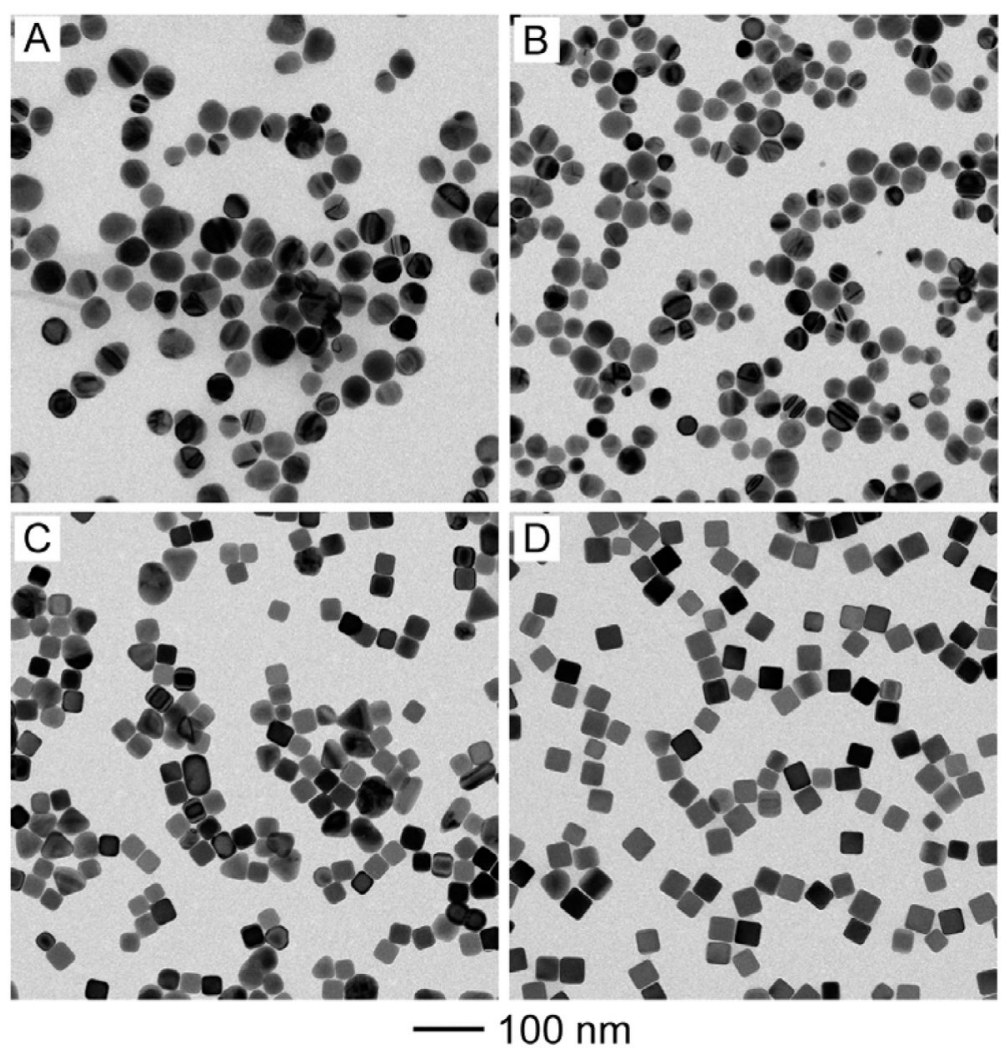

Figure 6.

TEM images of the products obtained using the same setup but under different gas atmospheres: (A) oxygen, (B) air, (C) nitrogen, and (D) argon. 
Table 1

Summary of the yield of Ag nanocubes in reactions under different gas atmospheres.

\begin{tabular}{ccc}
\hline Gas atmosphere & Percentage of oxygen & Percentage of cubes in the product \\
\hline Oxygen & $100 \%$ & 0 \\
Air & $21 \%$ & 0 \\
Nitrogen & 0 & $81 \%$ \\
Argon & 0 & $97 \%$ \\
\hline
\end{tabular}

